\title{
Situações-problema Simuladas: uma Análise do Processo de Construção
}

\author{
Simulated Problem Situations: an Analysis of \\ the Construction Process
}

Aline Guerra Aquilante Roseli Ferreira da Silval Lucimar Retto da Silva de Avól Flávia Gomes Pileggi Gonçalves ${ }^{I}$ Mariza Borges Brito de Souza

\section{PALAVRAS-CHAVES \\ - Educação de Graduação em Medicina \\ - Aprendizagem Baseada em Problemas/métodos \\ - Avaliação de Programas \\ - Currículo.}

\section{RESUMO}

O curso de Medicina da Universidade Federal de São Carlos (UFSCar) tem seu currículo orientado por competência e utiliza metodologias ativas de aprendizagem. Nesse contexto, o presente estudo teve como objetivo identificar e avaliar a construção das situações-problema (SP) utilizadas na Atividade Curricular Situações-Problema do curso de Medicina da Universidade de 2006 a 2009. Para a realização da coleta de dados, foram selecionadas as 81 situações-problema trabalhadas da $1^{\underline{a}}$ à $4^{\underline{a}}$ série pelos estudantes da primeira turma. Para tanto, realizou-se um estudo de caso cuja análise descritiva buscou uma abordagem quali-quantitativa. As variáveis analisadas foram: gênero; idade; ocupação; religião; cor da pele; desfecho da situação; fases do processo saúde-doença; cenário; dimensões (biológica, psicológica e social); áreas de competência (cuidado individual, cuidado coletivo, gestão e educação); e áreas de conhecimento. O estudo mostrou que a forma como as SPs estão sendo elaboradas é coerente com a literatura, pois guarda relação com os perfis sociodemográfico e epidemiológico brasileiros. Além disso, a investigação identificou outras características relativas ao formato das SPS, como a redação em diálogos e a opção por situações curtas e longas.

The medical school curriculum at the Federal University in São Carlos (UFSCar) is competency-based and uses active learning methods. The current study aimed to identify and assess the construction of problem situations (PS) used in the Course on Problem Situations at the UFSCar School of Medicine from 2006 to 2009. For data collection, 81 problem situations were selected by the first class from the first to the fourth years. A case study was carried out with descriptive analysis and a qualitative-quantitative approach. The analytic variables were: gender, age, occupation, religion, skin color, outcome of the situation, phases of the health-disease process, scenario, dimensions (biological, psychological, and social), competency areas (individual care, collective care, management, and education), and knowledge areas. The study showed that elaboration of the problem situations is consistent with the literature, since they relate to Brazil's socio-demographic and epidemiological profile. The study also identified other characteristics related to the format, such as writing in dialogues and the choice between short and long situations. 


\section{CONTEXTO}

O curso de Medicina da UFSCar tem seu currículo orientado por competência, de modo a promover e acompanhar o desenvolvimento da prática profissional médica fundamentada em capacidades voltadas para o enfrentamento de situações relativas à ação do médico em um determinado contexto. São utilizadas metodologias ativas, tais como: a Aprendizagem Baseada em Problemas (ABP) e a aprendizagem com base na prática ${ }^{1}$.

O curso está estruturado em três ciclos educacionais organizados segundo a progressão do desempenho do estudante no domínio de cada área de competência, considerando-se graus crescentes de autonomia e o alcance de excelência. São eles: Integralidade do Cuidado I — primeiro e segundo anos letivos; Integralidade do Cuidado II - terceiro e quarto anos letivos; e Integralidade do Cuidado III — quinto e sexto anos letivos (internato).

Cada ciclo é dividido em três unidades educacionais: Unidade Educacional de Prática Profissional; Unidade Educacional Eletiva; e Unidade Educacional de Simulação da Prática Profissional. As unidades educacionais oferecidas a todos os estudantes são longitudinais e complementares, sendo estruturadas a partir dos desempenhos esperados para os estudantes nos cenários utilizados para o desenvolvimento da prática profissional.

Na Unidade Educacional de Prática Profissional (UEPP), o confronto direto com a realidade é o elemento fundamental do processo ensino-aprendizagem. Assim, sua realização se dá em cenários reais focalizando o desenvolvimento, em um determinado contexto, da prática profissional.

Na Unidade Educacional Eletiva (UEE), formada por atividades complementares desenvolvidas em períodos preestabelecidos na grade curricular, o estudante - com o apoio de um professor-orientador e aprovação do Conselho de Coordenação - elabora seu plano de ensino a partir de suas necessidades educacionais, à luz do perfil de competência, podendo desenvolvê-lo dentro ou fora da Universidade.

Na Unidade Educacional de Simulação da Prática Profissional (UESPP), os elementos disparadores do processo ensino-aprendizagem podem ser situações-problema de papel, dramatizações, filmes, situações simuladas da prática profissional e outros recursos que permitam a construção de saberes em cenários protegidos e controlados. Essa unidade é formada por duas atividades curriculares: (i) Estações de Simulação da Prática Profissional e (ii) Situações-Problema.

\section{USO DE SITUAÇÕES-PROBLEMA EM UNIDADES EDUCACIONAIS SIMULADAS}

No currículo do curso de Medicina da UFSCar, para o recorte de conteúdo das SPs, são consideradas, além das áreas de competência (Cuidado Individual, Cuidado Coletivo, Gestão do Cuidado, Organização do Trabalho em Saúde; e Educação), as situações que prevalecem no cenário onde os estudantes se inserem nas atividades da prática profissional. Para o Ciclo I (1 $1^{\underline{a}}$ e $2^{\underline{a}}$ séries), o cenário privilegiado é o da Atenção Básica, com foco nas Unidades de Saúde da Família (USFs). No Ciclo II ( $3^{\underline{a}}$ e $4^{\underline{a}}$ séries), além das USFs, amplia-se o cenário para as Unidades Básicas de Saúde (UBSs), onde a prática é realizada por área: Saúde da Mulher; Saúde da Criança; e Saúde do Adulto e Idoso. Além do cenário como foco, as SPs devem articular diferentes conteúdos disciplinares, possibilitando a integração básico-clínico - tanto do normal, quanto do patológico - , o desenvolvimento do raciocínio clínico epidemiológico e a articulação das dimensões biológica, psicológica e social do processo saúde-doença-cuidado.

Os conteúdos das SPs também são orientados pelos ciclos de vida (adulto e idoso, criança, mulher), agregando as áreas de conhecimento da saúde mental, da saúde coletiva e da educação, que são trabalhadas de forma transversal.

Quanto ao formato de construção da SP, além do usual, apresentado na forma de "caso", optou-se por construir situações em forma de diálogo entre pacientes e diferentes profissionais de saúde, com a finalidade de excluir o narrador onisciente, para que os próprios estudantes interpretem a situação. No Ciclo II, além desse formato também são ofertadas SPs que simulam a primeira consulta e o retorno, assim, as informações são apresentadas em dois momentos distintos. Outros disparadores são utilizados, tais como: artigos de jornal, prontuários, vídeos, palestras e filmes. As SPs podem ser longas ou curtas, diferenciando-se pelo tempo que os estudantes dispõem para processá-las nos pequenos grupos.

Embora se tenha os cenários de prática, os ciclos de vida, as áreas de competência, a integração básico-clínico e a articulação das dimensões biológica psicológica e social como referências gerais de construção, há a necessidade de uma maior interação entre os ciclos educacionais. Isso gerou a demanda para construção de uma matriz curricular cujo referencial foi construído a partir da intersecção de atributos, ações e contextos, sendo orientado pelo perfil profissional definido pelas Diretrizes Curriculares Nacionais para o curso de graduação em Medicina ${ }^{2}$ e pelo perfil de competência escolhido pela UFSCar.

\section{CONSTRUÇ̃̃O DE SITUAÇÕES-PROBLEMA: UMA REVISÃO DE LITERATURA}

No contexto da ABP, o disparador principal do processo ensino-aprendizagem é o problema ou situação-problema (SP). Para Jonassen³ , 1997, o problema é visto como uma situação desconhecida que gera uma necessidade que motiva os indiví- 
duos a buscarem uma solução. As soluções podem ser convergentes (únicas) ou divergentes (quando diferentes caminhos são possíveis). Existem distintas classificações para problema: bem ou mal definido; simples ou complexo; longos ou curtos; familiares ou desconhecidos. Essas variedades podem ser sintetizadas em três tipos de problema: os enigmas ou quebra-cabeças; problemas estruturados; e problemas desestruturados.

Os problemas do tipo enigma/quebra-cabeças são aqueles que possuem apenas uma resposta correta, em que todos os elementos necessários para a solução são conhecidos e é preciso utilizar lógica e processos algorítmicos para solucioná-los. Por não tratarem geralmente de casos condizentes com a maioria das situações da vida real, têm utilidade limitada no processo ensino-aprendizagem.

Já os problemas estruturados são dependentes de conteúdos e bases teóricas restritos, geralmente apresentados em uma etapa anterior. Por isso, também apresentam relevância limitada na solução de problemas cotidianos. Um roteiro para construir problemas desse tipo envolve a definição de uma área de conhecimento, o enunciado que dirige a tarefa, uma lista de pré-requisitos (conceitos necessários para entender o problema), princípios teóricos e descrição do problema.

Por fim, os problemas desestruturados são aqueles em que um ou mais aspectos não aparecem bem definidos, assim como os que fazem parte do cotidiano e da prática profissional. São problemas que permitem várias soluções — ou podem até não ter solução - e não se limitam a disciplinas e especialidades, exigindo a articulação de diferentes saberes para ser explica$\mathrm{do}^{3,4,5}$. Por estarem situados na prática cotidiana, eles são muito mais interessantes e significativos para os estudantes, que têm que analisar o contexto, definir a natureza do problema, relacioná-lo com a teoria e determinar quais informações e habilidades são necessárias para a sua compreensão.

O processamento de problemas desestruturados exige reflexão e questionamento de crenças e conhecimentos prévios. O processo de geração de soluções é criativo, dialético e não depende somente de experiências individuais, mas da colaboração de todos e também do aspecto atitudinal, evidenciando a presença do lado humano ao trazer o debate de princípios, valores, normas implícitas ou explícitas. Os problemas destinados a promover o pensamento crítico-reflexivo devem exigir a colaboração entre os estudantes; assim, estes devem sintetizar as ideias, tomar decisões e resolver as controvérsias, exercitando a tomada de soluções em grupo ${ }^{3,5,6}$. Como não há apenas uma resposta correta, opiniões divergentes emergem, fazendo com que os estudantes também desenvolvam a habilidade de fundamentar a solução e as explicações que encontraram. O desenvolvimento de argumentos convincentes envolve não apenas a cognição e a metacognição, mas também a natureza epistemológica dos processos. Para conseguir resolver as situações-problema de maneira satisfatória, é preciso reconhecer o tipo de problema, organizar o conhecimento com foco no cerne da questão e evitar que as características superficiais sejam distratores. A experiência auxilia na distinção entre foco central e elementos periféricos do problema ${ }^{3}$.

O problema deve proporcionar aos estudantes motivação e possibilidades de levantar hipóteses e questões de aprendizagem sobre os conceitos, explicações e teorias que envolvem o problema, os quais serão confrontados na busca de informações que cada estudante fará individualmente. Como na $\mathrm{ABP}$ o problema não é necessariamente para ser resolvido, e sim compreendido, o fato do problema ser "desestruturado" não constitui uma limitação para o processo de aprendizagem. No início, os problemas devem ser mais estruturados e trazer informações-chave que auxiliem os processos de pesquisa e raciocínio. Conforme os estudantes forem adquirindo maiores capacidades cognitivas e de conexões, os problemas vão sendo menos estruturados, exigindo mais raciocínio e buscas. Essa gradação é importante para que os graduandos consigam se adaptar de maneira confortável à $\mathrm{ABP}^{7}$.

Para a construção de problemas desestruturados são necessários alguns elementos, tais como: uma situação-problema; área de conhecimento; focos principais; casos: bases de conhecimento disponibilizadas aos alunos (por exemplo, casos reais, fragmentos de anotações de prontuários, artigos relacionados, vídeos); e apoio para a construção da argumentação ${ }^{3}$.

Os problemas estruturados ou desestruturados, na verdade, não são entidades dicotômicas, mas representam diferentes pontos de um continuum. Enquanto os do primeiro tipo estão enraizados na teoria do processamento das informações, os do segundo dialogam com o construtivismo ${ }^{3}$.

A modelagem do problema pode se dar a partir de mapas de interação causal que contêm uma representação visual de todas as partes essenciais do problema, bem como as relações delas em um nível de detalhamento e familiaridade que são apropriados para os estudantes. Primeiramente, é preciso identificar o perfil de competência profissional para que os problemas não se limitem a exercícios teóricos, e o conteúdo possa ser aplicado na prática profissional —, para depois elaborar as situações que irão envolver as habilidades necessárias.

Quem redige as situações-problema deve abordar prováveis problemas do mundo real, ou seja, os casos têm de ser autênticos, relevantes e representativos ${ }^{3,5}$. Uma fonte para isso são os profissionais que trabalham no contexto que está sendo retratado, podendo ser entrevistados para obtenção de 
informações. As situações-problema são mais realistas quando não trazem todos os elementos ou, então, apresentam informações irrelevantes, exigindo análise crítica. Os estudantes devem identificar as características do problema, o contexto, os conhecimentos prévios, o que precisam estudar e os meios efetivos e não efetivos de solucioná-lo³ .

Na fase preliminar, o professor deve elaborar a situação-problema considerando as condições de aplicabilidade, o material didático, os recursos humanos, as fontes de informação, o tempo, a avaliação e os objetivos propostos. Caso não tenha conhecimento acerca do assunto que será abordado, é interessante que se familiarize por meio de estudo e entrevista com especialistas. Para poder planejar adequadamente a atividade, também precisa conhecer a realidade, investigando as necessidades dos estudantes.

Na elaboração do plano, o professor tem de determinar objetivos, selecionar conteúdos, recursos necessários e procedimentos de avaliação. Na determinação dos objetivos pedagógicos, deve-se levar em conta não somente o domínio cognitivo, os saberes, mas também o desenvolvimento das capacidades de autonomia, negociação, comunicação e responsabilidade diante de situações concretas. Os conteúdos que podem ser abordados devem ser listados e organizados, contemplando diversas disciplinas, o que exigirá a ajuda de outros professores ou especialistas.

É preciso atentar para o risco de que, se os estudantes discutirem todos os elementos da situação-problema, as atividades podem se tornar improdutivas, e o grupo pode não chegar a lugar nenhum. Por isso, é interessante determinar os focos principais. A administração do tempo pode-se constituir um fator que determinará, de certa forma, os rumos da pesquisa/ busca, pois se o produto final for muito complexo ou trabalhoso, e o tempo disponível não permitir o aprofundamento que os alunos gostariam de ter, eles serão obrigados a fazer escolhas e recortes em função desse tempo ${ }^{6,8}$.

O conteúdo deve se adaptar bem ao conhecimento prévio dos estudantes, pois isso ajuda a mobilizar o que eles já sabem sobre o assunto. O caso não deve conter tantos dados, para que o processo não fique estagnado na separação do que é relevante ou não. Também devem ser evitadas informações sem propósito, que acabam tirando o foco do problema. $\mathrm{O}$ contexto deve ser adequado à prática profissional, pois, se o problema for muito artificial, a discussão ficará no questionamento de hipóteses da situação fictícia, gerando um resultado frustrante.

O conhecimento contextualizado presume ser mais acessível para uso posterior, já que as pistas situacionais que ativam o conhecimento são armazenadas dentro das mesmas estrutu- ras cognitivas. A construção das situações-problema deve deixar de ser exclusivamente centrada no conhecimento com base em experiências, devendo centrar-se no conhecimento com base em evidências ${ }^{9}$. O ideal é que os problemas não sejam construídos por especialistas, mas por profissionais que sejam capazes de articular todos os aspectos das situações. Deve-se evitar a tentação dos especialistas de incluir todos os aspectos do problema, transformando-o em um único caso gigante ${ }^{3}$.

Dutch $^{4}$ sistematiza alguns passos para construção de um problema: 1) listar os objetivos de aprendizagem para o problema; 2) definir o contexto do problema no mundo real - é importante procurar inspiração em revistas, jornais, em conversas com profissionais da área, para conseguir aplicações realistas dos conteúdos do problema; 3) estabelecer a estrutura do problema - nessa etapa, pode-se fazer as seguintes perguntas: Como será a aparência do problema? Que perguntas abertas é possível inserir? Qual será a estrutura do problema? Quantos períodos serão necessários para os estudantes concluírem o processamento do problema? Enquanto o problema é trabalhado, serão adicionadas informações aos estudantes em páginas subsequentes? Quais os recursos que os estudantes precisarão? Qual o produto os estudantes terão de elaborar?; 4) escrever um guia, detalhando como o problema deve ser trabalhado pelo professor.

Nessa mesma direção, Lambros ${ }^{10}$ também aponta etapas semelhantes que podem auxiliar na construção do problema, acrescentando que este não pode confundir os estudantes com muitas informações e detalhes. Segundo este autor, o problema deve se limitar a informações que serão utilizadas para a sua resolução, não devendo ser tão diretivo a ponto de informar o que os estudantes devem fazer. Situações mais detalhadas demandam maior tempo de processamento e busca de novas informações. Pequenas histórias requerem menos buscas e podem ser trabalhadas por completo em um ou dois encontros. Lambros sugere que os professores antecipem os recursos que os estudantes precisarão para processar o problema, por exemplo: capacitação para buscas, biblioteca, laboratórios, entrevistas e conferências com especialistas, visitas, etc.

Outro autor, Hung7, propõe um modelo denominado 3C3R para orientar a construção de problemas em todas as etapas da formação. $\mathrm{O}$ modelo compreende duas categorias de componentes: os centrais (conteúdo, contexto e conexão) e os processuais (research/pesquisa, raciocínio e reflexão).

Quanto aos aspectos relacionados ao conteúdo (3C), devem ser levados em consideração: 1) $\mathrm{O}$ alinhamento com as normas curriculares, ou seja, o primeiro passo é definir as metas e objetivos em conformidade com as diretrizes curriculares, seguidos pelos objetivos de aprendizagem, que con- 
tribuem para a adequação do alcance do problema e o equilíbrio da amplitude e profundidade do conteúdo; 2) O escopo de problemas, ou seja, delimitar uma hierarquia dos objetivos de aprendizagem para definir a amplitude e profundidade do problema. Os problemas que exigem raciocínio contribuem para o aprofundamento do estudo e motivam os estudantes.

O conteúdo é abordado no contexto em que será aplicado, uma vez que os conhecimentos e habilidades são apreendidos mais facilmente se apresentarem uma validade contextual, por meio da avaliação da relevância clínica/prática para os futuros profissionais, devendo ser tratada explicitamente no problema. Deve-se ter um cuidado com o excesso ou falta de contextualização, ou seja, problemas com muitas informações de contexto podem sobrecarregar os estudantes, enquanto os com poucas informações podem induzir ao erro. O grau de contextualização depende da aplicação futura de determinados conteúdos nos cenários de clínica/prática. A relevância e a proximidade do contexto influenciam na maneira como os estudantes se apropriam dos problemas, criando, assim, uma motivação.

A conexão é fundamental para o desenvolvimento da compreensão de como diferentes conceitos e variáveis se articulam em distintos contextos. Desse modo, é importante a abordagem de pré-requisitos, ou seja, os problemas devem ser sequenciados dos mais simples/básicos aos mais complexos/avançados, sendo que estes últimos devem se pautar em informações que já apareceram em problemas anteriores. Ter uma sobreposição de abordagem, pois nem sempre é possível traçar relações hierárquicas entre os conceitos; portanto, estes devem aparecer em vários problemas para que os alunos possam estudar cada um em relação a outros e construir um conhecimento mais complexo. Também uma abordagem multifacetada é necessária, ou seja, incorporar o mesmo conceito em vários problemas com diferentes contextos aumenta o entendimento e o repertório dos estudantes para resolver situações em seu domínio profissional, ajudando-os a perceber a natureza dinâmica dos conceitos. A aprendizagem de conceitos em apenas um tipo de problema pode dificultar a capacidade de transferir e lidar com problemas complexos, do mundo real.

O objetivo geral do princípio 3R é facilitar a participação significativa na investigação científica e nos processos de resolução eficaz e eficiente de problemas. Assim, a pesquisa (research) envolve a especificação do objetivo e a especificação do contexto. $\mathrm{O}$ raciocínio ocorre simultaneamente à pesquisa e envolve atividades cognitivas complexas. É imprescindível adequar os níveis de pesquisa e raciocínio do problema à fase de formação em que o estudante se encontra. Por fim, a refle- xão auxilia os estudantes não só a integrar o que aprenderam, mas a estimular a curiosidade para explorar o assunto com mais profundamente e desenvolver a autoaprendizagem. A reflexão é comumente realizada com a orientação dada pelos tutores, mas também pode ser incorporada nas situações-problema, para promover a independência do estudante e as habilidades metacognitivas.

Lima e Linhares ${ }^{11}$, com base na experiência acumulada de dez anos do currículo que utiliza ABP na Universidade Estadual de Londrina (UEL), propõem seis princípios para se escrever bons problemas. O primeiro diz respeito à realidade, uma vez que o problema deve ser factível, para que levante conhecimentos prévios, motive o estudo e também possibilite que o estudante resgate o que foi aprendido quando se deparar com algo semelhante na prática. Também esses autores recomendam a estreita vinculação com a literatura científica, sugerindo a utilização de fragmentos de textos científicos para o exercício do espírito crítico-reflexivo. É imprescindível que estejam afinados com os objetivos educacionais que se quer alcançar e que se mantenham dentro de limites compatíveis com os tempos disponíveis para a discussão e para o estudo, uma vez que o excesso de disparadores possibilita pouco aprofundamento do conteúdo. É necessário também valorar positivamente as questões congruentes com o currículo, evitando privilegiar especialistas ou generalistas, procurando não reforçar preconceitos com relação aos usuários e ao cuidado em saúde. Quanto à composição de um problema, os autores sugerem que o título não deve ser jocoso nem antiético; o texto deve ter disparadores relacionados ao objetivo de estudo que se quer alcançar e evitar gatilhos para outros temas que possam funcionar como distratores.

\section{METODOLOGIA}

Considerando o caráter inovador da utilização de situações-problema simuladas da prática profissional para a formação médica, foi realizada uma investigação com o propósito de evidenciar os princípios e os componentes para formulação dessas situações no contexto curricular. Este estudo, portanto, passa a ser um instrumento de comunicação e divulgação das potencialidades das SPs em unidades educacionais interdisciplinares, na formação de profissionais da saúde.

Pela natureza do objeto de estudo, esta investigação utilizou uma metodologia que se caracteriza como estudo de caso, do tipo descritivo, com abordagens qualitativa e quantitativa. O objetivo foi identificar e refletir acerca da construção das situações-problema utilizadas nas Unidades Educacionais de Simulação da Prática Profissional, na primeira turma do curso médico da UFSCar no período de 2006 a 2009. As situações-problema foram o objeto de análise deste estudo, totalizando 
81 situações-problema. Para a realização da coleta de dados foram selecionadas todas as situações-problema trabalhadas com os estudantes da primeira turma, sendo 22 da $1^{\text {a }}$ série, 18 da $2^{\underline{a}}$ série, 24 da $3^{\underline{a}}$ série e 17 da $4^{\underline{a}}$ srie. Para a análise dos dados, em um primeiro momento, foram determinadas as variáveis de análise das situações-problema, tais como: gênero; idade; ocupação (segundo as categorias de ocupação definidas pela ABEP - Associação Brasileira das Empresas de Pesquisa ${ }^{12}$ ); religião; cor da pele; desfecho da situação (alta, seguimento, óbito); fases do processo saúde-doença (promoção, prevenção, tratamento, reabilitação); condição (saúde ou doença); cenário (atenção primária, secundária, terciária); dimensões da situação (biológica, psicológica, social); áreas de competência (cuidado individual, cuidado coletivo, gestão do cuidado, educação); áreas do conhecimento (segundo disciplinas do curso médico). No que se refere à análise quantitativa das situações-problema, ela foi sistematizada em um grande quadro, e as informações foram analisadas a partir de frequências absolutas e porcentagens dispostas em tabelas. A partir dessa análise, puderam ser feitas algumas considerações qualitativas quanto às situações-problema.

Essa investigação faz parte do projeto A implantação de um currículo de Medicina: uma análise sob a óptica de seus diferentes atores, do grupo de pesquisa "Educação para Profissionais de Saúde", o qual foi submetido a todos os procedimentos de autorização institucional e recebeu parecer favorável do Comitê de Ética e Pesquisa em Seres Humanos da UFSCar.

\section{ANÁLISE E INTERPRETAÇÃO DOS DADOS}

Problemas bem elaborados são cruciais para o sucesso da ABP. Eles funcionam como um organizador de conteúdos e conhecimentos, conferindo-os um contexto, o que torna a aprendizagem motivadora ${ }^{7}$. O contexto pressupõe a presença de distintas variáveis. Nas situações-problema deste estudo foram analisadas as variáveis gênero, ciclo de vida, área de conhecimento, ocupação, religião, cor da pele, desfecho, processo saúde-doença, condição de saúde-doença, níveis de atenção, área de competência, e dimensões biológica, psicológica e social.

Ao todo, foram trabalhadas 81 situações-problema (SPs) de 2006 a 2009 pela Turma I. Em relação ao gênero dos personagens, houve um equilíbrio entre masculino $(53,1 \%)$ e feminino $(44,4 \%)$, sendo que em $2,5 \%$ não constava ou não se aplicava essa categoria.

Acerca da distribuição por ciclo de vida, há uma concentração de casos relacionados à vida adulta de 20 a 59 anos de idade (56,9\%), o que já era esperado por ser o ciclo mais longo. Em seguida, aparece a adolescência, entre 10 e 19 anos de idade, com $14,8 \%$, e $11,1 \%$ de casos de idosos a partir de 60 anos.
As menores frequências foram de 1 a 9 anos de idade (8,7\%) e menores de 1 ano de idade (3,7\%). Comparando os dados das SPs com os do Censo Populacional de 2000, há uma equivalência na distribuição pelas diferentes faixas etárias. Segundo o IBGE ${ }^{13}$, a maior concentração aparece na faixa etária de 20 a 59 anos (51,3\%), a segunda maior na de 10 a 19 anos de idade $(20,8 \%)$, seguida por crianças de 0 a 9 anos de idade $(19,4 \%)$ e idosos $(8,6 \%)$.

A distribuição da ocupação dos personagens, realizada de acordo com o Critério Padrão de Classificação Econômica da $\mathrm{ABEP}^{12}$, pode ser observada na tabela 1. A maioria dos personagens possuía ocupação com registro em carteira de trabalho $(29,7 \%)$, sendo classificados como empregado ou funcionário público. Esse percentual está aquém dos dados de estudos sobre o tipo de vínculo empregatício, que aponta $42,4 \%$ dos trabalhadores com carteira assinada em $2006^{14}$. Em seguida, $16,0 \%$ eram estudantes. Chama a atenção o pequeno percentual de desempregados, visto que os dados do DIEESE ${ }^{15}$ de desemprego em São Paulo, em 2009, foram de 13,8\%.

\section{TABELA 1:}

Distribuição dos personagens das situações-problema segundo ocupação.

\begin{tabular}{l|r|r}
\hline \multicolumn{1}{c|}{ Ocupação dos personagens } & n & \multicolumn{1}{c}{$\%$} \\
\hline Aposentado & 5 & 6,2 \\
Autônomo & 7 & 8,6 \\
Dona de casa & 3 & 3,7 \\
Empregado & 19 & 23,5 \\
Estudante & 13 & 16,0 \\
Funcionário público & 5 & 6,2 \\
Não possui ocupação & 3 & 3,7 \\
Trabalhadores manuais sem capacitação & 8 & 9,9 \\
NA (não se aplica) & 9 & 11,1 \\
Desempregado & 3 & 3,7 \\
Estudante/trabalhador manual sem & 1 & 1,2 \\
capacitação & 5 & $\mathbf{6 , 2}$ \\
NC (não consta) & $\mathbf{8 1}$ & $\mathbf{1 0 0 , 0}$ \\
\hline Total & &
\end{tabular}

Com relação ao aspecto religioso, as SPs permitiram que os estudantes tivessem aproximação com diferentes crenças, com predomínio da religião católica $(21,0 \%)$ e da umbanda $(13,6 \%)$, embora na maioria dos casos $(43,2 \%)$ não constasse informações sobre a religião. Segundo dados do Censo $2000^{13}$, há predomínio da religião católica, havendo coerência com as situações apresentadas. O alto percentual de personagens da religião umbanda nas situações, diferente dos dados do IBGE, 
deu-se ao fato de terem sido trabalhadas várias pessoas de uma mesma família em situações distintas.

Sobre a cor da pele, $48,1 \%$ das SPs não faziam referência a essa categoria. $\mathrm{O}$ que predominou foi a cor branca $(35,8 \%)$, e entre negros e pardos o percentual foi de $12,4 \%$.

Quanto aos desfechos, 66,7\% corresponderam a casos de pacientes em tratamento. Em $27,2 \%$, a história não apresentava um desfecho claro e foi trabalhado apenas um caso de óbito, no $1^{\circ}$ ano do curso.

No que se refere ao processo saúde-doença, em $63,0 \%$ das situações-problema se trabalhou exclusivamente o tratamento; em 21,0\%, tratamento e prevenção associados e, em 6,2\%, associação entre promoção e prevenção. Em um único caso $(1,2 \%)$ foi abordada promoção de saúde exclusivamente e em 6,2\%, apenas prevenção. $\mathrm{O}$ enfoque em tratamento continua predominante, apesar das propostas atuais de formação de profissionais de saúde preconizarem a ampliação das dimensões do cuidado, principalmente na promoção à saúde e prevenção de doenças ${ }^{2,16}$. Porém, vale a pena ressaltar que, nesse currículo, a atividade de Prática Profissional, desde o $1^{\circ}$ ano do curso, tem uma forte presença dos aspectos preventivos e de promoção à saúde 1 .

$\mathrm{Na}$ categoria condição saúde e doença, 77,8\% dos casos apresentavam algum tipo de patologia, enquanto que em somente $14,8 \%$ foram trabalhadas condições de saúde. Ao todo, 79 condições de saúde foram trabalhadas, sendo 24 na $1^{\mathfrak{a}}$ série, 13 na $2^{a}$ série, 24 na $3^{\underline{a}}$ série e 18 na $4^{\underline{a}}$ série. Três desses diagnósticos foram abordados nas situações-problema em mais de uma série, sendo todos de alta prevalência e relevância. São eles: pneumonia aguda; obesidade; e gestação normal, sendo este último abordado em todas as séries. O formato em espiral do currículo possibilita que, a cada encontro com o tema, este seja abordado de forma mais ampla.

Observando a proporção representada pela distribuição dos diagnósticos abordados na $1^{1}$ série, conforme a divisão proposta pelo Código Internacional de Doenças (CID-10), nota-se uma concentração de temas catalogados no capítulo XXI (20,8\%), no qual há situações que influenciam o estado de saúde dos indivíduos e que não caracterizam doença, o que condiz com a proposta pedagógica do curso. Na 2ª série, observou-se uma maior variedade na distribuição dos diagnósticos abordados, sendo que doenças dos tecidos osteomusculares $(15,4 \%)$ e neoplasias $(15,4 \%)$ foram preferencialmente abordadas, as quais apresentam grande prevalência na população adulta e idosa. Na $3^{\underline{a}}$ série, houve uma preferência nítida por doenças do aparelho geniturinário $(20,8 \%)$ e por situações em que havia sinais, sintomas e achados laboratoriais sem diagnósticos fechados (20,8\%), de forma a se privilegiar as discussões a respeito de diagnósticos diferenciais. Na $4^{\underline{a}}$ sé- rie, verificou-se uma maior concentração de doenças do aparelho digestório $(22,2 \%)$, sobre o qual só havia sido abordada uma situação anteriormente no curso, e também de casos de transtornos mentais e comportamentais $(16,7 \%)$, de doenças do sistema nervoso $(11,1 \%)$ e do aparelho respiratório $(11,1 \%)$. De uma forma geral, houve uma distribuição ampla de doenças, sendo que as situações mais abordadas apresentaram relação com as prevalências dessas doenças em nosso meio. Dentre elas, citam-se as doenças endócrinas, nutricionais e metabólicas, que estiveram presentes em 10,1\% dos diagnósticos, dentre as quais se inclui a diabetes mellitus, doença de alta prevalência em nosso país (7,6\% da população geral), causa importante de morbidade e também de mortalidade que, juntamente com a obesidade, constitui a causa mais frequente dos óbitos por problemas cardiovasculares ${ }^{17,18}$. Outro grupo de doenças priorizado nas SPs foram as doenças do aparelho respiratório $(11,4 \%)$, nas quais se incluem as pneumonias agudas e as pneumonias crônicas, sendo que tais doenças constituem a quinta causa de falecimentos no Brasil e, dentre essas, a pneumonia é a segunda mais frequente, com 35.903 mortes em 2005 , sendo $8,4 \%$ delas em menores de 5 anos e $61,0 \%$ nos maiores de 70 anos $^{19}$. As doenças do aparelho geniturinário como um todo também apresentaram frequência aumentada nos estudos $(10,1 \%)$, embora quase metade dessas situações fosse relacionada com afecções renais e pouco mais que a metade em aparelho genital feminino. Nas quatro séries, temas que envolvem problemas na gestação, parto e puerpério, no período neonatal, más-formações e doenças do ouvido e da apófise mastoide não foram abordados.

Acerca do contexto em que os casos ocorreram, houve predominância da Atenção Primária à Saúde (70,4\%), seguida da Atenção Secundária (13,6\%). Esses dados são coerentes com o cenário de inserção dos estudantes na Atividade Curricular de Prática Profissional da $1^{\underline{a}}$ à $4^{\underline{a}}$ série, pois estavam inseridos em Unidade de Saúde da Família (USF) e Unidade Básica de Saúde (UBS). Nesta última, os estudantes passaram em esquema de rodízio pelas áreas de Saúde da Mulher, Saúde da Criança e Saúde do Adulto e Idoso.

Em 98,7\% dos casos foi abordada a dimensão biológica, sendo $28,4 \%$ das situações com foco exclusivamente biológico. Em 40,7\% apareceram associadas as dimensões psicológica e social, 22,2\% somente articulada à social, e 7,4\% juntamente à psicológica. Apenas uma situação trabalhou a dimensão social isoladamente $(1,3 \%)$. Esses resultados são condizentes com as expectativas, considerando que o aspecto biológico deve permear a quase totalidade das SPs e, quando possível, estar associado a situações psicológicas e socioculturais que possam interferir no processo saúde-doença. 
Quanto às áreas de competência, de acordo com o PPP, era previsto que os problemas abordassem tanto os Cuidados às Necessidades Individuais de Saúde quanto os Cuidados às Necessidades Coletivas de Saúde, a Organização do Trabalho em Saúde, a Gerência do Cuidado e a Educação. Porém, dos casos trabalhados, 75,3\% abordaram exclusivamente o cuidado individual, e cerca de $20,0 \%$ associaram o cuidado individual a outra área de competência. Somente 3,6\% das SPs focalizaram exclusivamente o cuidado coletivo, educação ou gestão (organização do trabalho). Mesmo sabendo que a prática tem seu foco principal no cuidado individual, nesta análise esse foco se mostrou excessivo, aparecendo em $95,0 \%$ das SPs trabalhadas.

Houve predomínio de situações na área de conhecimento de Saúde do Adulto e Idoso, sendo que 45,7\% das SPs abordaram exclusivamente essa área. Em seguida, vieram Saúde da Mulher (19,8\%) e Saúde da Criança (18,5\%). As áreas de conhecimento Saúde Coletiva e Saúde Mental, que geralmente apareceram associadas a outras áreas, constituíram o foco exclusivo em apenas 3,7\% e 1,2\% dos casos, respectivamente. As áreas de Educação e Saúde da Família e Comunidade só apareceram associadas a outras áreas, representando somente 2,4\% das SPs. É esperado que a área de Saúde do Adulto e Idoso seja a mais trabalhada, devido à maior concentração dessa faixa etária na população brasileira. Contudo, as proporções foram muito desiguais na distribuição das situações por área de conhecimento.

A análise dos conteúdos foi realizada a partir do quadro que traz a relação de disciplinas necessárias ao desenvolvimento da competência médica ${ }^{1}$. Nos casos em que não ficaram claras as áreas de conhecimento nem os conteúdos, utilizou-se a classificação "indefinido". Quando estava evidente a área de conhecimento e ela não fazia parte da relação de disciplinas, classificou-se como "outras áreas de conhecimento".

No quadro 1, é possível verificar que em todas as séries foram elaboradas situações-problema que permitiam a abordagem de conteúdos relativos à anatomia, anatomia patológica, fisiologia e biofísica, gestão em saúde, imunologia e semiologia clínica. As áreas de informática em saúde, inglês instrumental em saúde e parasitologia não foram contempladas, e outras apareceram em apenas alguns anos (biologia molecular, ética e bioética, genética médica e medicina legal). A não abordagem de algumas áreas de conhecimento é justificada, pois as SPs têm objetivos de aprendizagem especialmente relacionados ao desenvolvimento do domínio cognitivo e não são a única estratégia de aprendizagem, ocorrendo de forma concomitante e complementar às atividades curriculares de estações de simulação e prática profissional, que podem e devem abordar as áreas aqui não contempladas. Verificou-se também a necessidade de um melhor detalhamento das ementas, para que fiquem claros os conteúdos que devem ser abordados. Sugere-se, também, uma readequação do quadro das disciplinas, uma vez que vários conteúdos presentes nas SPs, que são importantes para a formação médica, pertenciam a áreas de conhecimento que não estão presentes na relação.

\section{QuAdRO 1}

Disciplinas abordadas nas situações-problema da $1^{\underline{a}}$ turma do curso de Medicina da UFSCar, segundo a série.

\begin{tabular}{|c|c|c|c|c|}
\hline Disciplinas & $1^{\mathrm{a}}$ série & 2a série & $3^{\mathrm{a}}$ série & $4^{\mathrm{a}}$ série \\
\hline Anatomia & $\mathrm{S}$ & $\mathrm{S}$ & $\mathrm{S}$ & $\mathrm{S}$ \\
\hline Anatomia patológica & $\mathrm{S}$ & $\mathrm{S}$ & $\mathrm{S}$ & $\mathrm{S}$ \\
\hline Biologia molecular & $\mathrm{S}$ & $\mathrm{N}$ & $\mathrm{N}$ & $\mathrm{N}$ \\
\hline Bioquímica & $\mathrm{S}$ & $\mathrm{S}$ & $\mathrm{S}$ & $\mathrm{N}$ \\
\hline Embriologia humana & $\mathrm{N}$ & $\mathrm{S}$ & $\mathrm{N}$ & $\mathrm{S}$ \\
\hline Ética e bioética & $\mathrm{N}$ & $\mathrm{N}$ & $\mathrm{N}$ & $\mathrm{S}$ \\
\hline Farmacologia terapêutica & $\mathrm{S}$ & $\mathrm{N}$ & $\mathrm{S}$ & $\mathrm{S}$ \\
\hline Fisiologia e biofísica & $\mathrm{S}$ & $\mathrm{S}$ & $\mathrm{S}$ & $\mathrm{S}$ \\
\hline $\begin{array}{l}\text { Educação e pesquisa em } \\
\text { saúde }\end{array}$ & $\mathrm{N}$ & $\mathrm{S}$ & $\mathrm{S}$ & $\mathrm{S}$ \\
\hline Gestão em saúde & $\mathrm{S}$ & $\mathrm{S}$ & $\mathrm{S}$ & $\mathrm{S}$ \\
\hline Genética médica & $\mathrm{N}$ & $\mathrm{S}$ & $\mathrm{N}$ & $\mathrm{N}$ \\
\hline Histologia & $\mathrm{S}$ & $\mathrm{S}$ & $\mathrm{N}$ & $\mathrm{N}$ \\
\hline Imunologia & $\mathrm{S}$ & $\mathrm{S}$ & $\mathrm{S}$ & $\mathrm{S}$ \\
\hline Informática em saúde & $\mathrm{N}$ & $\mathrm{N}$ & $\mathrm{N}$ & $\mathrm{N}$ \\
\hline $\begin{array}{l}\text { Inglês instrumental em } \\
\text { saúde }\end{array}$ & $\mathrm{N}$ & $\mathrm{N}$ & $\mathrm{N}$ & $\mathrm{N}$ \\
\hline Medicina legal & $\mathrm{S}$ & $\mathrm{N}$ & $\mathrm{N}$ & $\mathrm{N}$ \\
\hline Microbiologia & $\mathrm{S}$ & $\mathrm{S}$ & $\mathrm{N}$ & $\mathrm{S}$ \\
\hline Parasitologia & $\mathrm{N}$ & $\mathrm{N}$ & $\mathrm{N}$ & $\mathrm{N}$ \\
\hline Semiologia clínica & $\mathrm{S}$ & $\mathrm{S}$ & $\mathrm{S}$ & $\mathrm{S}$ \\
\hline $\begin{array}{l}\text { Outras áreas de } \\
\text { conhecimento }\end{array}$ & $\mathrm{S}$ & $\mathrm{S}$ & $\mathrm{S}$ & $\mathrm{S}$ \\
\hline Indefinido & $S$ & $S$ & $\mathrm{~S}$ & $\mathrm{~N}$ \\
\hline
\end{tabular}

Nota: $\mathrm{N}$ - não, $\mathrm{S}$ - sim

\section{CONSIDERAÇÕES FINAIS}

As opções deste modelo de currículo e da metodologia da ABP estão afinadas com a multidisciplinaridade, proporcionando a integração das ciências básica e clínico-epidemiológica. As situações de papel, trabalhadas nos pequenos grupos de aprendizagem, garantem que todos os estudantes sejam expostos aos mesmos problemas e contemplam essas integrações, dando assim um caráter sistematizado ao currículo. Dessa forma, a SP tem-se mostrado um excelente disparador para o processo de aprendizagem. 
A ABP não se limita à solução de problemas, mas os utiliza para o aumento da apreensão do conhecimento, não só de caráter cognitivo, mas também de desenvolvimento de capacidades de autonomia, negociação, comunicação e responsabilidade diante das situações.

A forma como as SPs estão sendo elaboradas no curso de Medicina da UFSCar são coerentes com os princípios e critérios definidos na literatura e guardam relação com o perfil sociodemográfico e epidemiológico brasileiro. Elas são do tipo estruturado e desestruturado, em um continuum, permitindo ao estudante o desenvolvimento de capacidades reflexivas ao longo de sua formação. Além disso, também se somam outras características às SPs, como a redação em formato de diálogo e a opção por situações curtas e longas.

Para além da revisão da literatura e análise das SPs do curso de Medicina da UFSCar, este estudo possibilitou o redimensionamento de categorias para construção de situações-problema tais como: gênero; idade; ocupação; religião; cor da pele; desfecho (tratamento, recuperação, morte); condição e fases do processo saúde-doença; cenários de atenção à saúde; dimensões (biológica, psicológica e social); áreas de competência e áreas de conhecimento e conteúdos - que permitem a ampliação do olhar do estudante em relação ao processo saúde-doença-cuidado.

\section{REFERÊNCIAS}

1. Universidade Federal de São Carlos. Coordenação do Curso de Medicina. Caderno do Curso de Medicina. São Carlos; 2007.

2. Brasil. Ministério da Educação. Conselho Nacional de Educação. Câmara de Educação Superior. Resolução CNE/CES no 4, de 07 de novembro de 2001. Institui Diretrizes Curriculares Nacionais do Curso de Graduação em Medicina. Diário Oficial da União, Brasília, 9 nov. 2001. Seção 1, p. 38.

3. Jonassen DH. Instructional design models for well-structured and III-structured problem-solving learning outcomes. Educ Tech Res Dev..1997;45(1):65-94.

4. Dutch B. Problem-Based Learning Clearinghouse. PBL Clearing House: Problem Writing Guide. [online]. Newark, DE: University of Delaware; 2001 [acesso em $16 \mathrm{dez}$. 2009]. Disponível em: <http://www.udel.edu/pblc/problems $>$.

5. Weiss RE. Designing problems to promote higher-order thinking. New Directions for Teaching and Learning 2003;2003(95):25-31.

6. Pietrocola M, Pinho AJ, Pinheiro TF. Prática interdisciplinar na formação disciplinar de professores de ciências. Investigações em ensino de ciências. [periódico na internet].
2003. [acesso em 22 fev. 2010]; ;8(2):131-152. Disponível em: $<$ http://www.if.ufrgs.br/public/ensino/>.

7. Hung W. The 3C3R Model: a conceptual framework for designing problems in PBL. The IJPBL. 2006;1(1):55-77.

8. Schmitz C, Alves Filho JP. Ilha de racionalidade e a situação problema: o desafio inicial [online]. Trabalho apresentado no IX Encontro de Pesquisa em Ensino de Física; 26-30 out. 2004 [acesso em 08 dez. 2009];Jaboticatubas, MG. Disponível em: <http://www.sbf1.sbfisica.org.br/eventos/epef/ ix/sys/resumos/T0106-1.pdf>.

9. Dolmans DHJM, Snellen-Balendong H, Wolfhagen IHAP, Van der Vleuten CPM. Seven principles of effective case design for a problem-based curriculum. Med Teach. 1997;19(3):185-9.

10. Lambros A. And the problem is. In: Lambros A. Problem-based learning in middle and high school classrooms: A teacher's guide to implementation. Thousand Oaks, CA: Corwin Press; 2004 p.42-54.

11. Lima GZ, Linhares REC. Escrever bons problemas. Rev Bras Educ Med. 2008;32(2):197-201.

12. Universidade Estadual Paulista. Faculdade de Medicina de Botucatu. Projeto Viver Bem. DABEP. Critério padrão de classificação econômica Brasil/2008 [acesso em 12 maio 2010]. Disponível em: http://www.viverbem.fmb.unesp. br/docs/classificacaobrasil.pdf

13. Instituto Brasileiro de Geografia e Estatística. Censos demográficos [online]. Acesso em 12 maio 2010]. Disponível em: http://www.ibge.gov.br/brasil_em_sintese/populacao02.htm.

14. Hallak J, Namir K, Kozovits L. Setor e emprego informal no Brasil: Análise dos resultados da nova série do sistema de contas nacionais - 2000/2006 [online]. [acesso em 12 maio 2010]. Disponível em: http://www.ie.ufrj.br/datacenterie/pdfs/seminarios/pesquisa/texto1310.pdf.

15. Departamento Intersindical de Estatística e Estudos Socioeconômicos. Taxa de desemprego total, por sexo [online]. acesso em 12 maio 2010]. Disponível em: http:/ / turandot. dieese.org.br/icv/TabelaPed?tabela $=5$.

16. Brasil. Ministério da Saúde. Ministério da Educação. Pró-saúde: programa nacional de reorientação da formação profissional em saúde. Brasília: MS, MEC; 2005.

17. World Health Organization. Definition, Diagnosis and Classification of Diabetes Mellitus and its complications; 1999.

18. Silvestre JA. Hospitalizações SUS/1997. Dez principais causas por enfermidades - Idosos CID-9. Brasília: Ministério da Saúde; 1998.

19. Corrêa RA, Lundgren FLC, Pereira-Silva JL, Frare e Silva RL, Cardoso AP, Lemos ACM et al. Diretrizes brasilei- 
ras para pneumonia adquirida na comunidade em adultos imunocompetentes - 2009. J Brasileiro Pneumologia. 2009;35(6):574-601.

\section{CONTRIBUIÇÃO DOS AUTORES}

Aline Guerra Aquilante trabalhou na concepção do artigo, na pesquisa bibliográfica, na coleta, análise e interpretação dos dados e na redação final do texto. Roseli Ferreira da Silva trabalhou na concepção do artigo, na coleta, análise e interpretação dos dados e na redação final do texto. Lucimar Retto da Silva de Avó participou da concepção do artigo, da pesquisa bibliográfica, da coleta dos dados e da redação inicial do texto. Flávia Gomes Pileggi Gonçalves trabalhou na coleta, análise e interpretação dos dados. Mariza Borges Brito de Souza participou da concepção do artigo e da redação final do texto.
CONFLITO DE INTERESSES

Declarou não haver.

\section{ENDEREÇO PARA CORRESPONDÊNCIA}

Aline Guerra Aquilante

Departamento de Medicina - UFSCar.

Rod. Washington Luís, km 235

Monjolinho - São Carlos

CEP. 13565-905 SP

E-mail:aline@ufscar.br 